\title{
A Data Forwarding Scheme Based on Delaunay Triangulation for Cyber-Physical Systems
}

\author{
Junhai Luo, Yijun Cai, and Changping Zhong \\ School of Electronic Engineering, University of Electronic Science and Technology of China, Chengdu 611731, China \\ Correspondence should be addressed to Junhai Luo; junhai_luo@uestc.edu.cn
}

Received 18 November 2012; Accepted 17 March 2013

Academic Editor: Igor Andrianov

Copyright ( 2013 Junhai Luo et al. This is an open access article distributed under the Creative Commons Attribution License, which permits unrestricted use, distribution, and reproduction in any medium, provided the original work is properly cited.

\begin{abstract}
Cyber-physical system (CPS) cooperates with physical processes, computing, communication, and control (3C) into multiple levels of information processing and operation management to streamline and fortify the operation of physical systems. Due to the unique characteristics, such as unpredictable node mobility, low node density, lack of global information and network intermittent connectivity, an algorithm for data forwarding in CPS is a considerably difficult and challenging problem, and there is no good solution to it in existing works. In this paper, we propose a fully-fledged data forwarding algorithm tailored to the CPS environment. The proposed protocol, called data forwarding based on Delaunay triangulation (DFDT), takes into account the computational geometry based on Delaunay triangulation to form a few triangle communities according to nodes' connectivity. Data in a community are forwarded to other nodes once a node comes into this community to increase the data delivery ratio. DFDT achieves a good performance by data gathering and sending data to other nodes with higher probability of meeting the link. An extensive simulation has been performed to validate the analytical results and to show the effectiveness of our approach compared with the three existing popular data forwarding algorithms.
\end{abstract}

\section{Introduction}

As an emerging field, cyber-physical system (CPS) [1-3] research has gained increasing research attention, which cooperates physical processes, computing, communication and control (3C) into multiple levels of information processing and operation management to streamline and fortify the operation of physical systems.

CPS is composed of and interconnected by various components (e.g., sensors, monitors, controllers, actuators, and embedded computers) through communication networks. Information collected by sensors from physical infrastructure in CPS is adapted to cyber components with using communication links to intelligently feedback control the physical components, which is computed by information processing center, such as cloud computing. There is typically no oneto-one correspondence between the elements of the two networks, which complicates the understanding of their interaction. Critical infrastructure systems reliant on intelligent monitoring and control are among the most important CPS and also prime examples of pervasive computing systems, as they exploit computing to provide "anytime, anywhere" transparent services. While the added intelligence offers the promise of increased utilization, its impact must be assessed, as unrestricted information control can actually lower the reliability of existing infrastructure systems. Compared with the traditional networks, CPS owns characteristics, such as self-judgment, self-control and self-regulation, node mobility, and network intermittent connectivity.

CPS has gained increasing attention in a wide range of application domains, such as transportation system, modern airplane, nuclear power plant, highway traffic, embedded medical devices, oil refiner, power grid, and health care. Such system must be able to operate safely, dependably, securely, efficiently, and in real-time, potentially highly uncertain or unstructured environments. CPS is expected to have great technical, economic, and social impacts in the near future.

The convergence of sensing, communication, processing, control, and coordination in CPS poses enormous challenges. The aspects seem particularly challenging [4]: distributed and concurrent interactions between cyber and physical 
systems; strong QoS requirements including real-time fault tolerance, network intermittent connectivity, sensing node autonomy, security and effective data sampling, and sensing and forwarding.

The data gathering and forwarding schemes in the traditional networks depend on a large number of nodes deployed densely with short communication range to form a connected end-to-end networks. It is unreasonable to assume end-toend connectivity in CPS, such as mobile ad-hoc networks (MANTs) [5], wireless sensor networks (WSNs) [6], and wireless mesh networks (WMNs) [7], satellite networks, military tactical networks, disaster response networks, and underwater networks. Physical components exchange buffered messages when they come into the communication range, and messages may be buffered and carried physically several times before ultimately being received. Therefore, traditional routing methods for data gathering and forwarding do not suit the environment of the intermittent connectivity CPS.

Thus, how to maintain not only a relatively longer system lifetime but also a higher information delivery ratio with the lower transmission overhead and data delivery delay has become the most important problem to solve. Different techniques have been proposed for data gathering and forwarding. Researchers in those papers [8-10] have contributed some schemes for the data forwarding in the interrupted networks, but they failed to make contributions to the research on the mobility nodes deployed in CPS.

In this paper, we propose a fully fledged data forwarding algorithm tailored to CPS environment. The proposed protocol, called data forwarding based on Delaunay triangulation (DFDT), takes into account the computational geometry for nodes deployment and wireless signal coverage to increase the data delivery ratio. DFDT algorithm is discussed and thoroughly analyzed. DFDT achieves a good performance by gathering and sending data to other nodes with higher probability of meeting the link. An extensive simulation campaign has been performed to validate the analytical results and to show the effectiveness of our approach compared with the three existing popular data forwarding algorithms, namely DT, epidemic, and SpWT. Simulation results show that DFDT does not only achieve a relatively low data forwarding overhead but also gets the higher message forwarding ratio with lower data forwarding delay.

The remainder of the paper is organized as follows. We summarize the recent related work on CPS in addition to existing node deployment for networks in Section 2. In Section 3, we develop a theoretical framework for data forwarding in CPS. Based on the framework, we develop a detailed Delaunay triangulation algorithm for data forwarding in CPS in Section 4. The performance analysis and simulation of data forwarding based on Delaunay triangulation in CPS are presented in Section 5, and we conclude our work in Section 6.

\section{Related Work}

Our work is inspired and motivated by some research efforts on delay tolerant mobile sensor networks (DTMSNs). Due to the wide range, the novel field and the limited space of related work in CPS, we only briefly introduce the work closely related to our proposal.

The research on data forwarding originates from epidemic (flooding) scheme [11], which floods the entire network. Epidemic is modeled from the manner in which diseases spread in the population. When two nodes are in range, they trade summary vectors containing the unique identifiers of the stored messages and use them to determine which messages to transfer. The vectors contain both currently and previously carried messages, preventing a node from receiving the same message twice. In essence, epidemic Routing is a flooding algorithm, with each node sending messages to all of its neighbors. This, in turn, means that it requires very little information about the network, which makes it useful for a wide range of scenarios. Its main weaknesses are the heavy use of storage space and radio transmissions.

Some other data forwarding strategy approaches concentrate on trading off data complexity versus increasing the likelihood of data delivery. To limit the number of data single copy routing schemes, allow only one copy of the data to be present in the network at a time [12]. One of the basic approaches for data forwarding is a direct transmission (DT) scheme, where each source node keeps its data until it comes into direct contact with the respective destination nodes. More specifically, assume that the generated data message is inserted into a first come first serve (FCFS) queue. Whenever the node meets the other node, it transmits the data messages in its queue to the other node. A node receives or transmits any data messages of other sensors. The sensors are usually activated and deactivated periodically. The node performs sensing and generates one data message upon waking up in each period. Under this scheme, only one data transfer is made per delivered data, incurring minimal data passing. However, in intermittently connected networks, such an approach may produce low delivery ratios and has an unbounded delivery delay.

To address overhead problems in flooding, different forms of controlled flooding have been proposed. One of the basic approaches for data forwarding is a present spray and wait (SpWt) [13]. In this method, there are two phases: spray phase which spreads the copies and wait phase which performs direct transmissions. In the SpWt, a total of $L$ copies of a message are initially spread to other "relay" nodes. If the destination is not found in this phase, each of the nodes carrying a copy of the message will perform direct transmission. In essence, SpWt is a type of controlled flood [14]. No mobility or connectivity information regarding the nodes in the network is assumed to be known for this scheme to work.

Later studies develop data forwarding strategies to approach the performance of epidemic routing with lower forwarding cost, which is measured by the number of data copies created in the network. The data forwarding metric, which measures the nodes' capability of contacting others, is generally independent from the data forwarding strategies mentioned above. Various metrics can be applied to the same forwarding strategy for different performance requirements. 


\section{Delaunay Triangulation for CPS}

3.1. Delaunay Triangulation. At first, we review the definition of Delaunay triangulation. We assume that all wireless nodes are given as a set $p$ of $n$ nodes in a two-dimensional space. Each node has some computational power. We also assume that there are not four nodes of $p$ that are cocircular. A triangulation of $p$ is a Delaunay triangulation if the circumcircle of each of its triangles does not contain any other nodes of $p$ in its interior. A triangle is called the Delaunay triangle if its circumcircle is empty of nodes of $p$ inside [15]. Delaunay triangulation has some properties that make it unique [16]. Firstly, external Delaunay edges constitute the boundary of the convex hull of $p$. Secondly, all circumcircles of Delaunay triangulation are empty. Routing in the Delaunay triangulation will ensure that the packet will reach its destination. And then, in Delaunay triangulation the expected number of node degree is less than or equals 6 for all network sizes. So, each node has to store the information about 6 adjacent nodes.

3.2. Question Analysis. In the CPS network, it has its unique characteristics. (1) Indeterminacy: an end-to-end path between the source and the destination may only exist for brief and unpredictable periods of time. With the increased use of wireless mobile devices, many new network applications fall into this category, such as wildlife tracking, military networks, and disaster recovery and emergency response systems; (2) node mobility: the node moves randomly based on RWP model among different communities to link up data interaction of senor nodes, and the CPS topology is also highly dynamic because of node mobility (3) Spare density: node density is much lower in CPS than that of transitional networks.

Due to those characteristics of CPS described above, traditional proactive and reactive routing schemes fail to work in CPS scenario. However, this does not mean that the packets cannot be delivered to the destination. Because of node mobility, different links come up and down over time, enabling nodes to achieve eventual delivery through a storedforward approach, which uses buffers to hold the message until the next link comes up in the end-to-end path due to node mobility. A necessary condition for this approach to work is the existence of an end-to-end path between source and destination in a combined connectivity graph formed by overlapping connectivity graphs over a time interval.

After deploying nodes in a target area, there is no established network among these nodes. The nodes do not have any idea about the location of the other nodes and the architecture of the network. We can take advantage that the nodes in CPS have ability of self-configuring, selfcontrol, and self-organizing, and consider that the intermittent network connectivity and data gathering and forwarding between the communities are connected by the mobile nodes, which have the ability of joining communities quickly while being sure of their own locations in certain communities.

3.3. CPS Model. In this paper, we suppose that all wireless nodes given as a set $p$ of $n$ nodes in CPS are randomly

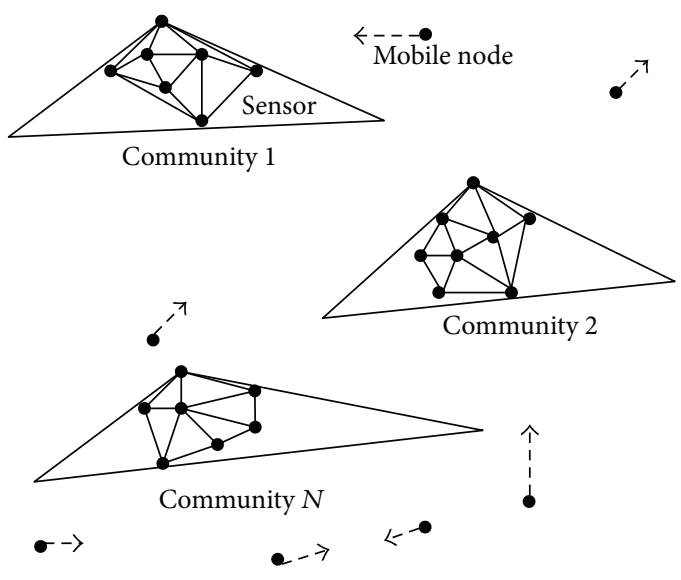

FIGURE 1: Illustration of network model and communities.

deployed in a square area with size of $M * M$, and meanwhile, this area is divided into several two-dimensional communities (each connective region formed by some nodes in CPS is called a community [17]) areas of different sizes. Because the communication range of nodes is finite, as shown in Figure 1, where the whole CPS is not connective and divided into several communities, nodes in the same community can directly communicate with each other. All the nodes are divided into three communities, and some nodes which belong to any communities in the model are called loner nodes in Figure 1, which are capable of continuously moving in different communities of our system model to ferry data forwarded from other nodes. Furthermore, we also assume some of the following characteristics.

(i) The node mobility model is mainly used to describe the location and velocity of the node and its variation, which is an important tool to describe the encounter characteristics between the nodes. Data forwarding and transfer depend on meeting opportunities during node mobility, and node mobility model determines the probability of encounters between nodes and encounter time distribution, which is the basis of designing data forwarding algorithm. In order to study the data forwarding of CPS nodes, this paper first assumes CPS node connectivity scene. A certain number of nodes make random movement in the area according to random waypoint (RWP) mobility model [18], which is still one of the most widely used mobility models for the wireless scenario simulations, and they enter the divided community areas randomly. As a typical mobility model, RWP is also widely used in the research of mobile wireless networks, such as MANETs, WSNs, and WMNs.

(ii) All the nodes have the same maximum transmission range $R(m)$, the same maximum node speed $V(\mathrm{~m} / \mathrm{s})$, and the same computational power.

(iii) Each node can only communicate directly with other nodes in the same community or the adjacent community area (sharing the same line or angle is called 
adjacent), and all the nodes are all surely in their own communities.

(iv) All the nodes have the attached extra location devices, such as a low-power GPS or some other way, to easily attain their locations [19]. The resources used to location initialization and broadcast the location of the sensor node have been ignored [20,21].

\section{Data Forwarding Based on DFDT}

In this section, we propose a novel data forwarding based on DFDT in CPS environment. Considering the unique characteristics of CPS, DFDT aims to attain a high data delivery ratio with the minimum data delivery overhead and delay.

4.1. Community Structure. In CPS environment, due to the node random mobility and the limitation communication range, the whole CPS is intermittent connectivity and divided into a certain number of stable nonconnective communities. As shown in Figure 1, only the nodes in the same communities can communicate, and two communities cannot communicate with each other without the connection of the mobile nodes.

In this paper, the first problem to be solved is how to divide a community. We firstly consider creating a triangle, which is big enough to surround all the nodes to form a community. In a random node set $p$, the sensor node on the farthest right among many nodes in coordinate $Y$ is considered as the vertex $p_{0}$ of this big enough triangle. Then, we also set another two vertexes, which are called $p_{1}$ and $p_{2}$, while making sure that the distance between $p_{1}$ and $p_{2}$ is far enough to be outside of any circumcircles of any three nodes.

How should we set another two vertexes of $p_{1}$ and $p_{2}$ ? Starting from sensor node $p_{0}$ and moving along coordinate $Y$ from top to bottom, we perform iteration detection nodes until we can never detect new targets, which means that no directly connective sensor nodes can be detected. And then starting from the smallest sensor node with the coordinate $Y$, we perform iteration detection along coordinate $X$ from left to right till no new targets can be detected. The iteration detection is performed according to the Friis free space propagation model [22], which assumes the ideal propagation condition that there is only one clear line-of-sight path between the transmitter and receiver to calculate the received signal power in free space at distance $d$ from the transmitter. The Friis transmission equation is as follows:

$$
P_{r}(d)=\frac{P_{t} G_{t} G_{r} \lambda^{2}}{(4 \pi)^{2} d^{2} L},
$$

where $P_{t}$ is the transmitted signal power, $G_{t}$ and $G_{r}$ are the antenna gains of the transmitter and the receiver, respectively. $L(L \geq 1)$ is the system loss, and $\lambda$ is the wavelength. It is common to select $G_{t}=G_{r}=1$ and $\lambda=1$ in the simulation experiment. The free space model basically represents the communication range as a circle around the transmitter. If a receiver is within the circle, it receives all packets. Otherwise, it loses all packets.
A node makes a decision on whether a target is detected or not based on its measurement on the intensity of target signal. The noise of a node obeys a Gaussian distribution with zero mean, and the target signal obeys a Gaussian distribution with nonzero mean. According to paper [23], the task of detection at node $i$ is to test the following two hypotheses:

$$
\begin{gathered}
H^{0}: p\left(z_{i} \mid H^{0}\right)=\frac{1}{\sqrt{2 \pi \delta}} \exp \left(-\frac{z_{i}^{2}}{2 \delta^{2}}\right), \\
H^{1}: p\left(z_{i} \mid H^{1}\right)=\frac{1}{\sqrt{2 \pi \delta}} \exp \left(-\frac{\left(z_{i}-P_{\mathrm{r}}(d)\right)^{2}}{2 \delta^{2}}\right),
\end{gathered}
$$

where $z_{i}$ represents the measurement at node $i . H^{0}$ and $H^{1}$ represent the hypothesis that the target is absent or present, respectively. $p\left(z_{i} \mid H\right)$ represents the detection probability at node $i$ under $H$ condition.

$$
u_{i}= \begin{cases}1, & \text { if } \frac{p\left(z_{i} \mid H^{1}\right)}{p\left(z_{i} \mid H^{0}\right)}>\varphi, \\ 0, & \text { otherwise. }\end{cases}
$$

Node $i$ decides a target is detected if its measurement is greater than a decision threshold, denoted as $\varphi$ as shown in formula (3): otherwise, it decides that no target is detected.

In this paper, one of the key design objectives of community and routing maintenance for computational complexity is to minimize the community (re-)configuration and node detection time. The computational cost for determining the node and community in the network is combinatorial due to the need to consider the combination of detection decisions from multiple nodes. It can be seen from (2) to (3) that the complexity of computing is $O\left(2^{N}\right)$, where $N$ is the number of sensors that participate in the community structure. In the future work, we will apply a fast numerical approximation method by a similar procedure [24].

4.2. Community Update. After the accomplishment of the discovery of the big triangle community $\Delta p_{0} p_{1} p_{2}$, denoted as $\Gamma$ for all nodes in CPS, we perform the community update based on Delaunay triangulation in order to create several small triangle communities where nodes can forward data directly. The algorithm for the community update is proposed in detail in Algorithm 1. As shown in Algorithm 1, for each node in $\Delta p_{0} p_{1} p_{2}$, its location initialization is performed and broadcasted. After the initialization, each sensor node has stored the information of location of itself and the ones' which can directly communicate with it. Then, starting from $p_{0}$, we connect all the sensor nodes which can directly communicate with each other and form a certain number of triangles community randomly and put these triangles in a community link one by one. After the establishment of the triangles, according to the rule that the circumcircle of this community does not contain any other sensor nodes in its interior, the iteration is performed for the optimization. Finally, the community update based on Delaunay triangulation of the senor node is reached. 


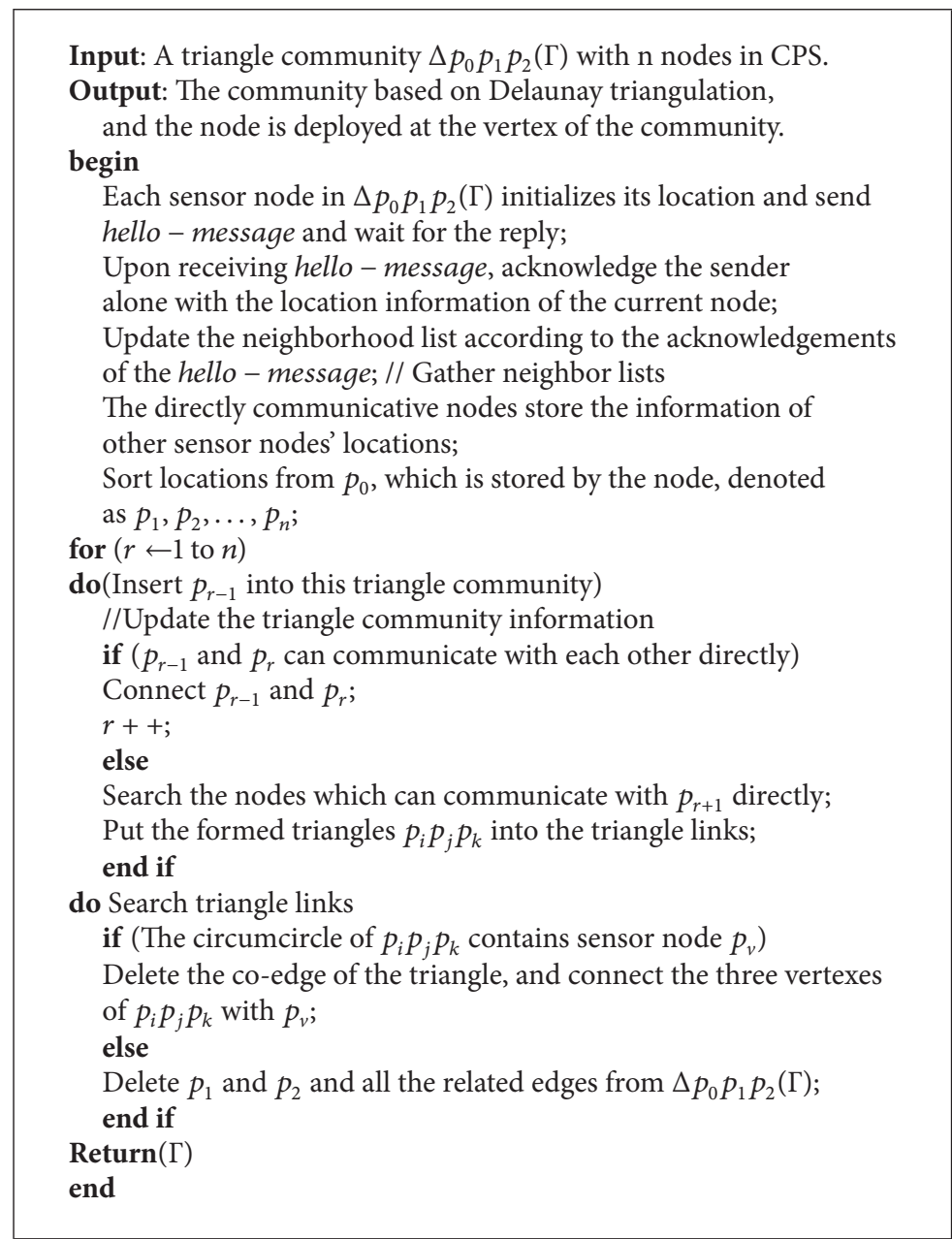

Algorithm 1: Pseudo-code of Community Update Based on Delaunay Triangulation.

4.3. Data Forwarding Based on DFDT. Different communities can communicate with each other based on the mobility nodes based on RWP model outside communities, which store, carry, and forward data to other communities and realize the whole CPS connectivity. When a node moves nearby a community, a node in the community detects this mobility node and forward data in its wireless range.

In our proposed scheme, we create a directed acyclic graph in each community, denoted as $D$, where each leaf of the graph corresponds to each triangle community which is stored in the present triangle community link, respectively. When a mobile node comes within one community, the community discovery based on Delaunay triangulation algorithm will work and divide new communities again. when the mobile node finds its own community according to the directed acyclic graph, it will communicate with the nodes on the vertexes of this community to avoid wasting energy when several nodes are collecting information and the mobile nodes are communicating with the further sensor nodes in the same hot area.

Then, let us make a explanation by the example of the node which contains three leaves as shown in Figure 2, when mobile node $p_{v}$ inserts $\Delta 2$, it will firstly connect with the three vertexes of $\Delta 2$ and form three new triangles, and they will correspond to three leaves. Then based on Delaunary triangulation method, four triangles finally are formed.

When mobile node $p_{v}$ moves into a certain community, firstly node $p_{v}$ quickly accomplishes the triangulation and is sure of its community's location. Node $p_{v}$ searches directed acyclic $D$ and locates the triangle communities containing the nodes, which node $p_{v}$ will communicate with. As illustrated in Figure 3 , we take node $n 1$ communication with node $n 3$ as the example. $n 1$ is in the triangle communities $\Delta 1, \Delta 2$, and $\Delta 3$, while $n 3$ is in the triangle communities $\Delta 4, \Delta 5$, and $\Delta 6$. Through the check for the adjacent triangle communities, we can find the two optimal paths, $n 1 \rightarrow n 2 \rightarrow n 3$ or $n 1 \rightarrow$ $n 4 \rightarrow n 3$. If $n 1$ forwards data to $n 3$ through the nodes in $\Delta 7$, it leads to the increase of the hop count, the delay of the data transmission, and causes the decrease of the availability ratio of nodes.

\section{Performance Evaluation}

In the previous section, we analyze data forwarding algorithms based on Delaunay triangulation in CPS environment 


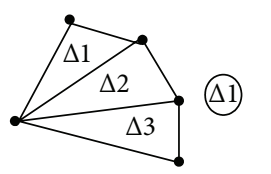

(a)

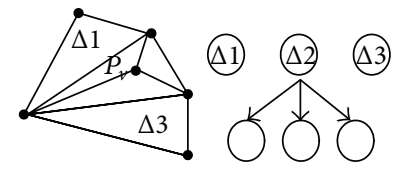

(b)

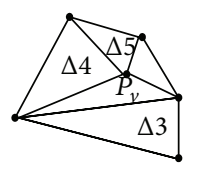

(c)
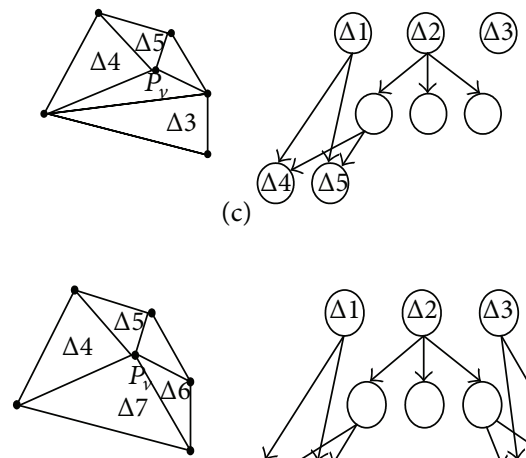

(d)

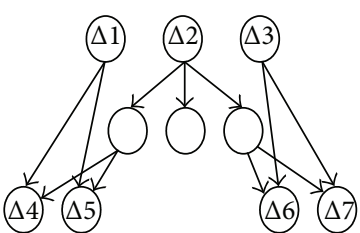

Figure 2: Changes Process of Node Joining.

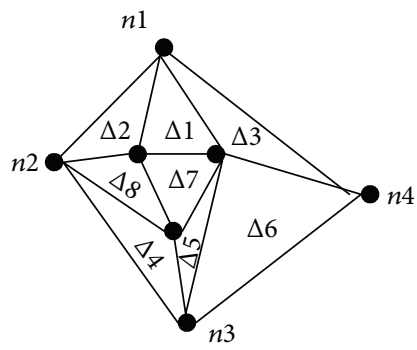

Community
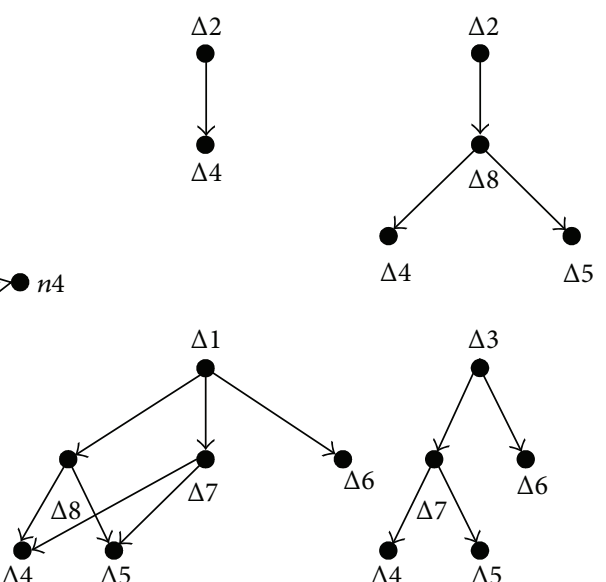

FIgURE 3: Transmitting Process from $n 1$ to $n 3$ Based on Community Search for Routing Path.

and show that it can dramatically reduce costs of data delivery. In this section, we select forwarding algorithms so as to include both well-known existing algorithms as well as algorithms that span a wide range of design choices for CPS.

5.1. Simulation Envionment. We work in the following setting: nodes generate messages over time; each message has a particular source and destination. At random times, nodes come into contact, meaning that they are capable of exchanging messages. Messages are transmitted in whole from node to node at time instants during node contact intervals of $50 \mathrm{~s}$, after which both nodes hold message replicas. In our simulations, we generate messages according to a Poisson process, and messages are transmitted with no transmission lag.

In our analysis, the metrics we are concerned with are (1) success ratio, which is the fraction of generated messages for which at least one replica is eventually delivered; (2) average delay, which is the average duration between a message's generation and the first arrival of one of its replicas at the destination; and (3) overhead, which is the number of replicas per generated message in the network. By "high performance," it means high success rate and low average delay. Other simulation parameters and their default values are summarized in Table 1.

5.2. Simulation Results. In this section, we compare the performance of the data forwarding approach with existing data forwarding schemes based on cumulative node data forwarding characteristics. We evaluate the performance of our approach in data delivery ratio and forwarding overhead measured by the number of data copies created in CPS, and each experiment is repeated 500 times for statistical convergence. The data delivery delay is not considered, as long as the data can be delivered on time. We compare our data forwarding metric with the following existing metrics based on cumulative contact characteristics.

5.2.1. Scenario A: Effect of Default Parameters. We compare the performance of the four data forwarding algorithms, 
TABLE 1: Simulation Parameters.

\begin{tabular}{lc}
\hline Parameter & Default value \\
\hline Network size $\left(\mathrm{m}^{2}\right)$ & $1000 * 1000$ \\
Number of sensor node & 50 \\
Max node moving speed $(\mathrm{m} / \mathrm{s})$ & 15 \\
Min node moving speed $(\mathrm{m} / \mathrm{s})$ & 10 \\
Initial energy of each sensor node (J) & 100 \\
Threshold vale of $\varphi$ & 8 \\
Energy for GPS communication per time (J) & 0.000025 \\
MAC layer type & IEEE 802.11 \\
Start time of sending data packets (s) & 500 \\
End time of sending data packets (s) & 525 \\
Interval of sending data packets (s) & 0.25 \\
Number of sending data packets & 100 \\
Packet buffer size (packet) & 800 \\
Data packet size (byte) & 512 \\
\hline
\end{tabular}

TAble 2: Simulation Results with Default Parameters.

\begin{tabular}{lcccc}
\hline & DT & Epidemic & SpWt & DFDT \\
\hline Success Delivery Ratio (\%) & 67.2 & 69.5 & 75.3 & 91.0 \\
Average Delivery Delay (s) & 1201.5 & 890.3 & 520.0 & 268.8 \\
Average Delivery Copies & 1 & 12.9 & 11.3 & 10.2 \\
\hline
\end{tabular}

namely, DT, epidemic, and SpWt, with the default parameters, as shown in Table 2.

As shown in Table 2, DFDT achieves average success delivery ratio of $91.0 \%$, which is much higher than the other three strategies, because the data forwarding strategy proposed in this paper effectively delivers data in a triangle community when meeting with a mobile node. We find that the SpWt is very unstable, because its performances vary significantly with different values of the replicas $l$ with different data forwarding schemes as presented in related work in Section 2. DT performs worst in terms of the delivery ratio, because it is difficult for nodes to forward data directly to the destination node. Furthermore, the delivery ratio of epidemic protocol is a little higher than that of DT. This stems the fact that data created by epidemic can be delivered to the destination nodes by different sensor nodes. So, the overall delivery ratio of epidemic outperforms DT.

At the same time, we are also interested in the data delivery delay and average overhead. As shown in Table 2, DFDT outperforms the other three schemes in terms of data delivery delay with lower delivery overhead. The number of average data copes of DT is always 1 , because nodes with the DT strategy ferry 1 data copy to other nodes within its communication range. Nodes with epidemic scheme forwarding data to other nodes by flooding have a longer average delivery delay than SpWt and DFDT. The average delivery overhead of SpWt also is affected by the number of replicas.

5.2.2. Scenario B: Accuracy Analysis of Default Parameters. Figure 4 shows the maximum error of the data forwarding of

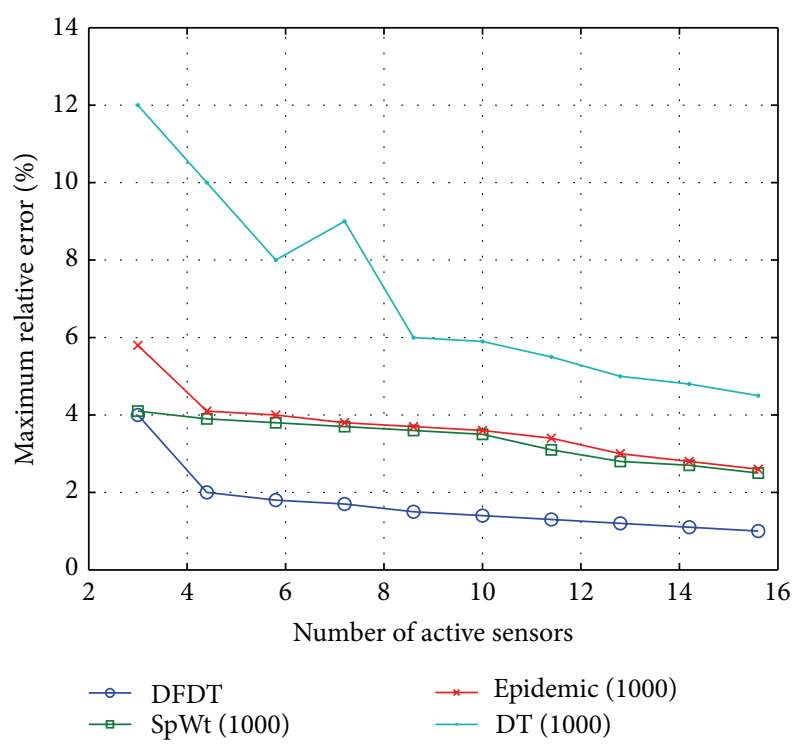

FIgURE 4: Maximum Errors of Data Forwarding Probabilities.

all sampling locations in the region. The number of sensors is varied from 1 to 15 , because calculating the exact system of more sensors is extremely slow due to the combinatorial complexity. Different curves of the methods in Figure 4 correspond to different numbers of experiments simulated. We can see that our proposed DFDT outperforms other methods even when the number of rounds in the latter is 1000. The error of DFDT drops quickly when the number of sensors increases. In particular, the maximum error is less than 1 percent when the number of active nodes is larger than 6.

5.2.3. Scenario C: Effect of Node Density. In this scenario, this experiment simulation depicts the impact of sensor node density by varying the total number of sensor nodes, because the connectivity of CPS is closely related to the density of sensor nodes. As shown in Figure 5, DFDT can always get higher data delivery ratio with the increase of the node density. This is reasonable, because the data delivery strategy is more aggressive in DFDT scheme. The delivery ratios of DT, SpWt, and epidemic vary slightly with the increase of sensor node density, which demonstrates that node density does not have significant impact on data delivery ratios in the other three algorithms.

With higher sensor node density, Figure 6 shows that the number of data copies in the DFDT scheme raises, and the number of average data copes of the DT is always 1 . In the DFDT protocol, more sensor nodes may enlarge the size of a community and make the total number of data in the community increase. Thus, more data can be delivered to a subscriber once the subscriber comes into contact with the community. Furthermore, more data in the buffer queue of nodes also make the number of reforwarded data increase as a result. In DT scheme, however, more sensors cannot meet the mobile nodes timely with more sensor nodes being deployed in the network. Thus, data in these sensors are unlikely to be 


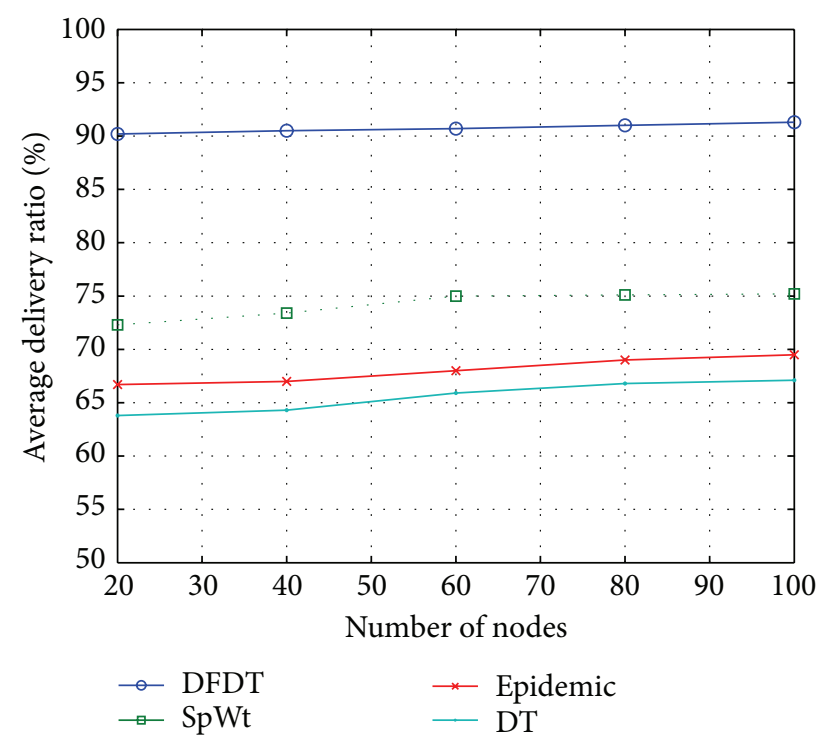

FIgURe 5: Average Delivery Ratio.

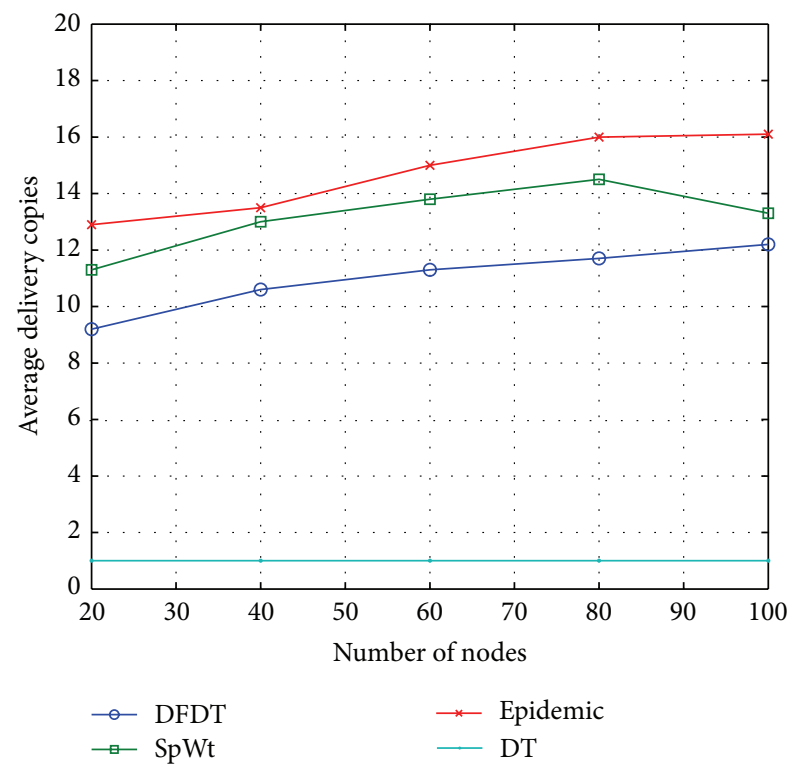

Figure 6: Average Delivery Copies.

received by nodes. This leads to the decrease of event copies in DT. As more copies can enlarge the opportunity to deliver the matched data to their nodes, the average data delivery delay decreases in DFDT protocol with the increase of sensor node density. In contrast, the data delivery delay decreases in DT protocol with higher sensor node density, as shown in Figure 7.

5.2.4. Scenario D: Effect of Transmission Range. This group of experiments illustrates the impact of node transmission range in CPS. As illustrated in Figure 8, DFDT achieves a higher delivery ratio than other three algorithms by varying each node wireless transmission range from $1 \mathrm{~m}$ to $10 \mathrm{~m}$ in

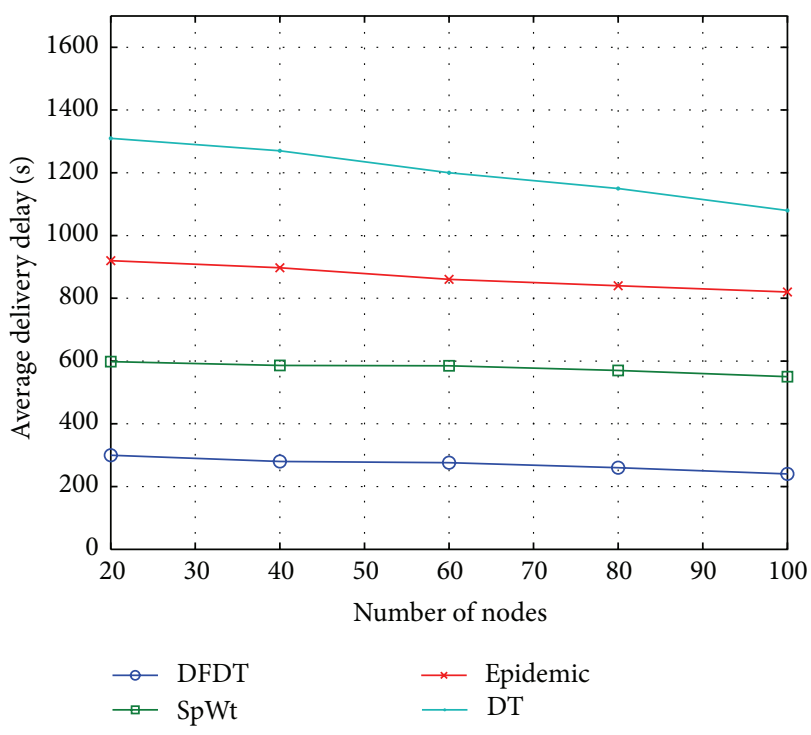

Figure 7: Average Delivery Delay.

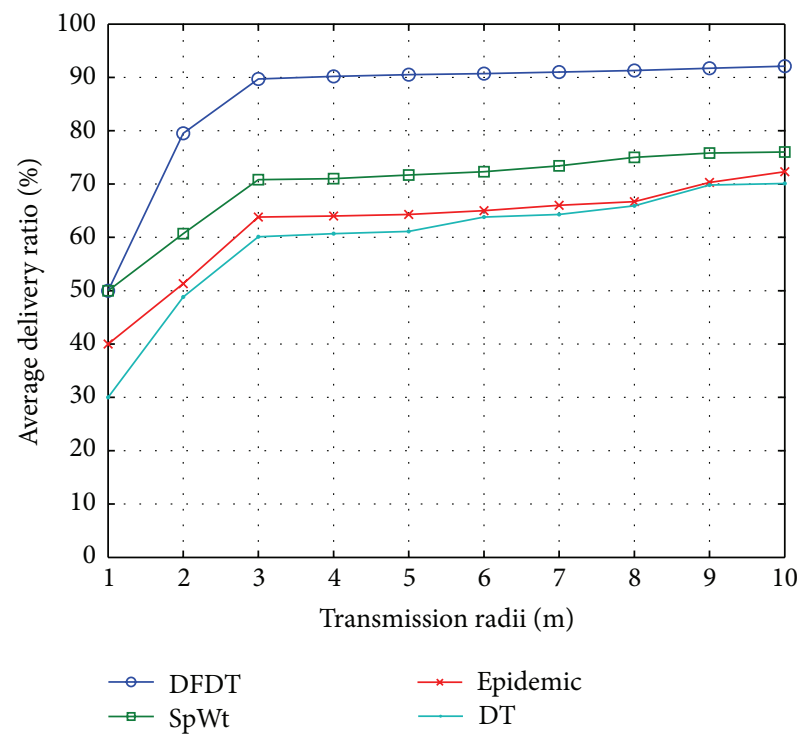

Figure 8: Average Delivery Ratio.

our simulations. With the increase of node transmission range, the delivery ratio increases in all strategies, because the nodes have higher probabilities of meeting others and increasing the probability of data relaying. However, with the very small transmission radii, the delivery ratios of all the strategies alter sharply by varying node transmission range from $1 \mathrm{~m}$ to $2 \mathrm{~m}$, which demonstrates that node transmission range has significant impact on data delivery ratios in CPS environment.

Figure 9 shows that, except the direct transmission, the number of average copies of other protocols increases with the transmission range. Figure 10 demonstrates that the average delay of each protocol decreases when transmission range becomes large. 


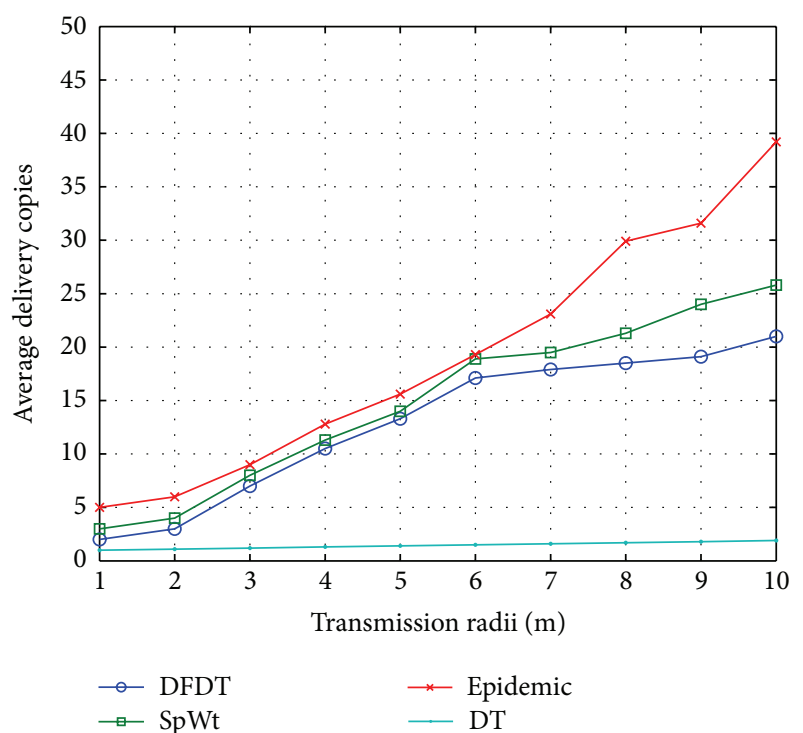

Figure 9: Average Delivery Copies.

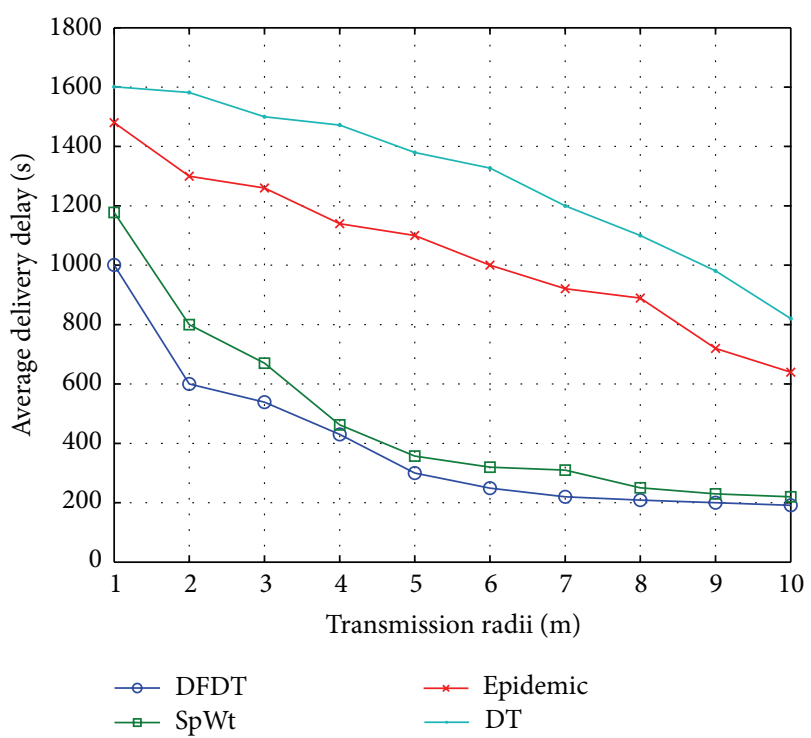

Figure 10: Average Delivery Delay.

\section{Conclusion}

In this paper, we propose a fully fledged data forwarding algorithm tailored to CPS environment. The proposed protocol, called data forwarding based on Delaunay triangulation (DFDT), takes into account the computational geometry for nodes deployment and wireless signal coverage to increase the data delivery ratio. The DFDT algorithm is discussed and thoroughly analyzed. DFDT achieves a good performance by data gathering and sending data to other nodes with higher probability of meeting the link with lower energy consumption. An extensive simulation campaign has been performed to validate the analytical results and to show the effectiveness of our approach compared with the three existing popular data forwarding algorithms, namely, direct transmission (DT), flooding, and epidemic. Simulation results show that DFDT does not only achieve a relatively low data forwarding energy consumption but also gets the higher message forwarding ratio with lower transmission overhead and data forwarding delay.

\section{Acknowledgments}

This research is supported by the National Natural Science Foundation of China under Grant no. 61001086 and the Fundamental Research Funds for the Central Universities Grant no. ZYGX2011X004.

\section{References}

[1] J. Chen, R. Tan, G. Xing, X. Wang, and X. Fu, "Fidelity-aware utilization control for cyber-physical surveillance systems," in Proceedings of the 31st IEEE Real-Time Systems Symposium (RTSS '10), pp. 117-126, December 2010.

[2] W. He, X. Liu, L. Zheng, and H. Yang, "Reliability calculus: a theoretical framework to analyze communication reliability," in Proceedings of the 30th IEEE International Conference on Distributed Computing Systems (ICDCS '10), pp. 159-168, June 2010.

[3] L. Parolini, N. Tolia, B. Sinopoli, and B. H. Krogh, "A cyberphysical systems approach to energy management in data centers," in Proceedings of the 1st ACM/IEEE International Conference on Cyber-Physical Systems (ICCPS '10), pp. 168-177, April 2010.

[4] L. Sha and J. Meseguer, "Software-intensive systems and new computing paradigms," in Design of Complex Cyber Physical Systems with Formalized Architectural Patterns, pp. 92-100, 2008.

[5] J. Luo, X. Liu, and M. Fan, "A trust model based on fuzzy recommendation for mobile ad-hoc networks," Computer Networks, vol. 53, no. 14, pp. 2396-2407, 2009.

[6] M. Keally, G. Zhou, and G. Xing, "Sidewinder: a predictive data forwarding protocol for mobile wireless sensor networks," in Proceedings of the 6th Annual IEEE Communications Society Conference on Sensor, Mesh and Ad Hoc Communications and Networks (SECON '09), pp. 538-546, June 2009.

[7] Y. Feng, H. Gong, M. Fan, M. Liu, and X. Wang, "A distanceaware replica adaptive data gathering protocol for delay tolerant mobile sensor networks," Sensors, vol. 11, no. 4, pp. 4104-4117, 2011.

[8] J. Zhu, M. Liu, J. Cao, G. Chen, H. Gong, and F. Xu, "CED: a community-based event delivery protocol in publish/subscribe systems for Delay Tolerant Sensor Network (DTSN)," in Proceedings of the 38th International Conference on Parallel Processing (ICPP '09), pp. 58-65, September 2009.

[9] J. M. Soares and R. M. Rocha, "CHARON: routing in lowdensity opportunistic wireless sensor networks," in Proceedings of the 2nd IFIP Wireless Days (WD '09), pp. 260-264, December 2009.

[10] H. Ahmadi, T. Abdelzaher, J. Han, N. Pham, and R. K. Ganti, "The sparse regression cube: a reliable modeling technique for open cyber-physical systems," in Proceedings of the IEEE/ACM 2nd International Conference on Cyber-Physical Systems (ICCPS '11), pp. 87-96, April 2011.

[11] J. M. Soares, M. Franceschinis, R. M. Rocha, W. Zhang, and M. A. Spirito, "Opportunistic data collection in sparse wireless 
sensor networks," Eurasip Journal on Wireless Communications and Networking, vol. 2011, Article ID 401802, 2011.

[12] Y. Wang and H. Wu, "Delay/fault-tolerant mobile sensor network (DFT-MSN): a new paradigm for pervasive information gathering," IEEE Transactions on Mobile Computing, vol. 6, no. 9, pp. 1021-1034, 2007.

[13] T. Spyropoulos, K. Psounis, and C. S. Raghavendra, "Spray and wait: an efficient routing scheme for intermittently connected mobile networks," in Proceedings of the ACM SIGCOMM Workshops: Conference on Computer Communications, pp. 252259, August 2005.

[14] E. J. JebaJothi, V. Kavitha, and T. Kavitha, "Contention based routing in mobile ad hoc networks with multiple copies," CoRR abs/1005.4009.

[15] D. Satyanarayana and S. V. Rao, "Constrained delaunay triangulation for ad hoc networks," Journal of Computer Systems, Networks, and Communications, vol. 2008, Article ID 160453, 10 pages, 2008.

[16] M. B. Haider and K. Sugihara, "Almost Delaunay triangulation routing in wireless sensor networks," in Proceedings of the 10th International Conference on Computer and Information Technology (ICCIT '07), pp. 1-7, December 2007.

[17] J. Zhu, J. Cao, M. Liu, Y. Zheng, H. Gong, and G. Chen, "A mobility prediction-based adaptive data gathering protocol for delay tolerant mobile sensor network," in Proceedings of the IEEE Global Telecommunications Conference (GLOBECOM '08), pp. 730-734, December 2008.

[18] M. Abdulla and R. Simon, "The impact of the mobility model on delay tolerant networking performance analysis," in Proceedings of the 40th Annual Simulation Symposium (ANSS '07), pp. 177184, March 2006.

[19] F. Xu and M. Liu, "A motion tendency-based adaptive data delivery scheme for delay tolerant mobile sensor networks," in Proceedings of the 28th IEEE Conference on Global telecommunications (GLOBECOM '09), pp. 3978-3983, 2009.

[20] A. Deshpande, Z. Ives, and V. Raman, "Adaptive query processing," Foundations and Trends in Databases, vol. 1, pp. 1-140, 2007.

[21] L. Wang and A. Deshpande, "Predictive modeling-based data collection in wireless sensor networks," Lecture Notes in Computer Science, vol. 4913, pp. 34-51, 2008.

[22] 2011, http://www.isi.edu/nsnam/ns/doc/node218.html.

[23] G. Xing, X. Chang, C. Lu et al., "Efficient coverage maintenance based on probabilistic distributed detection," IEEE Transactions on Mobile Computing, vol. 9, no. 9, pp. 1346-1360, 2010.

[24] D. P. Arvind, A. Arasu, B. Babcock et al., "Stream: the stanford stream data manager," IEEE Data Engineering Bulletin, vol. 26, no. 2003, 2003. 


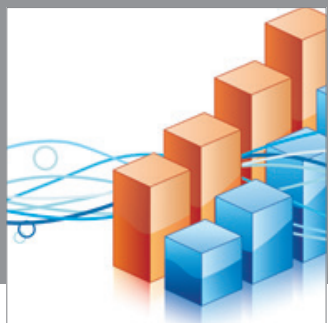

Advances in

Operations Research

mansans

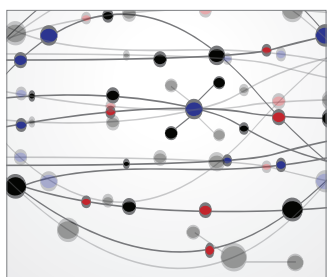

The Scientific World Journal
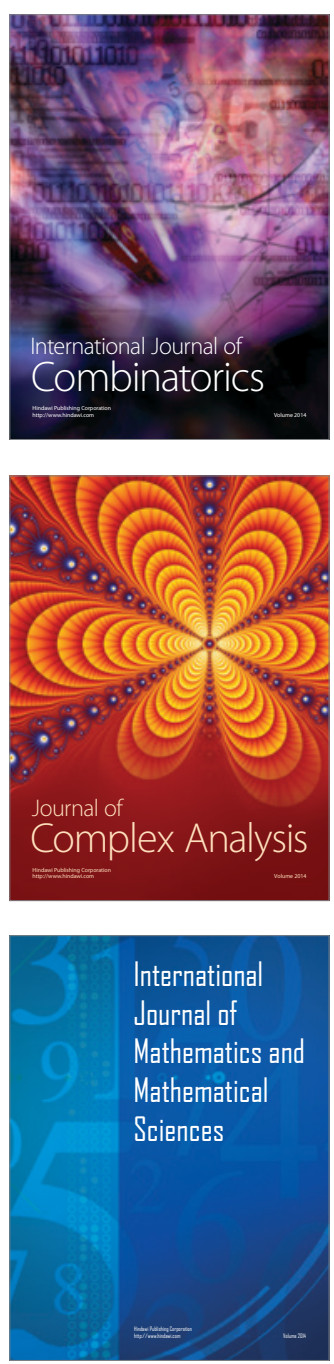
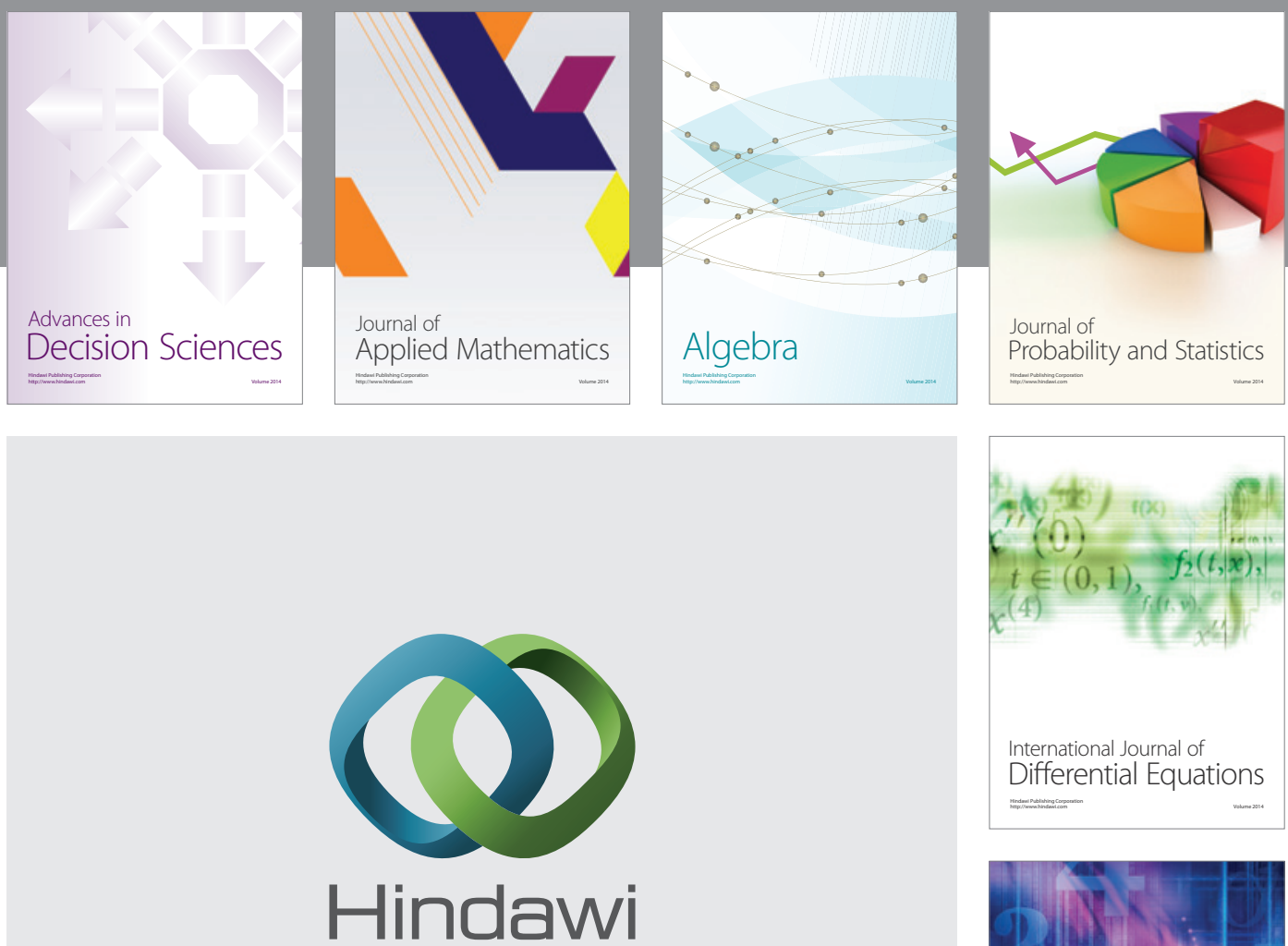

Submit your manuscripts at http://www.hindawi.com
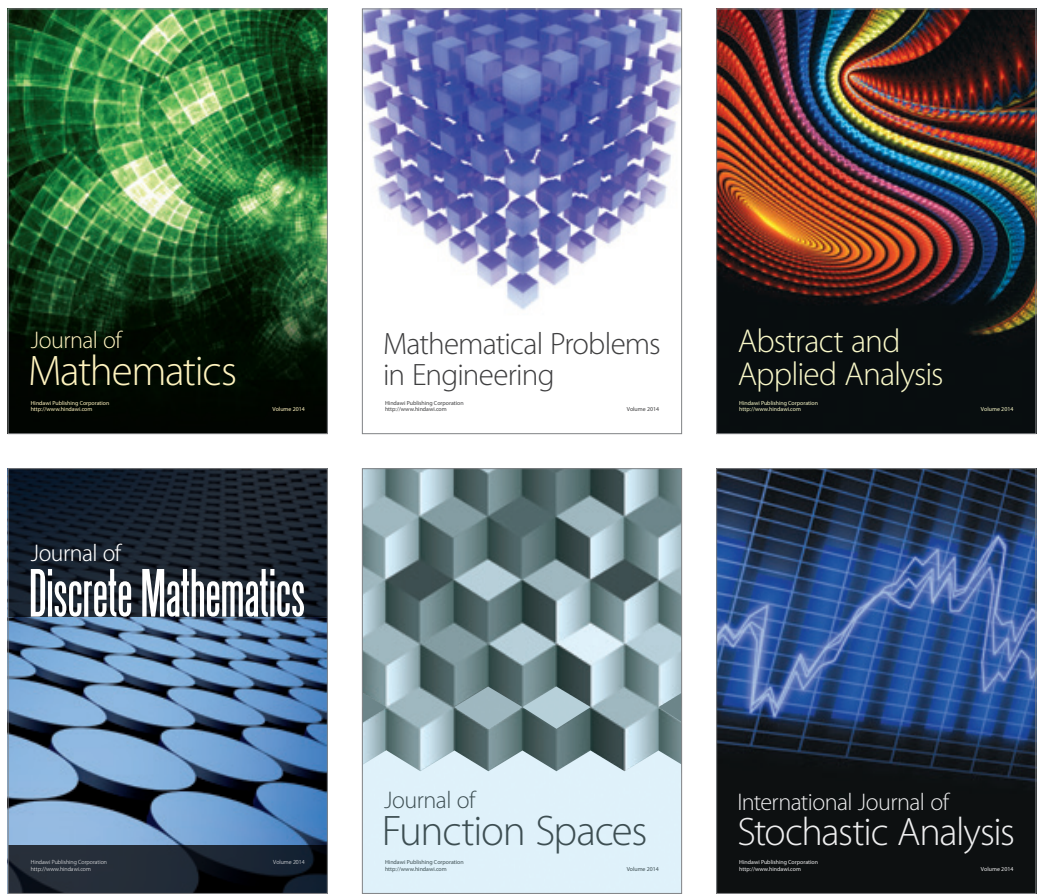

Journal of

Function Spaces

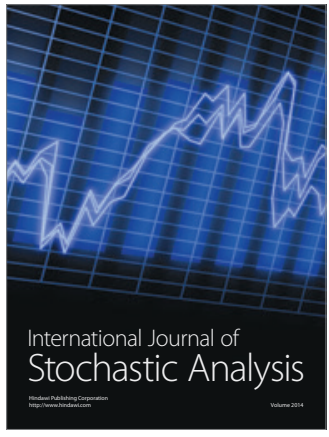

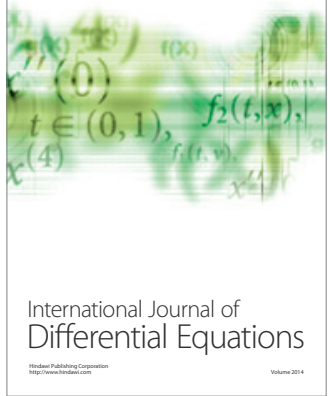
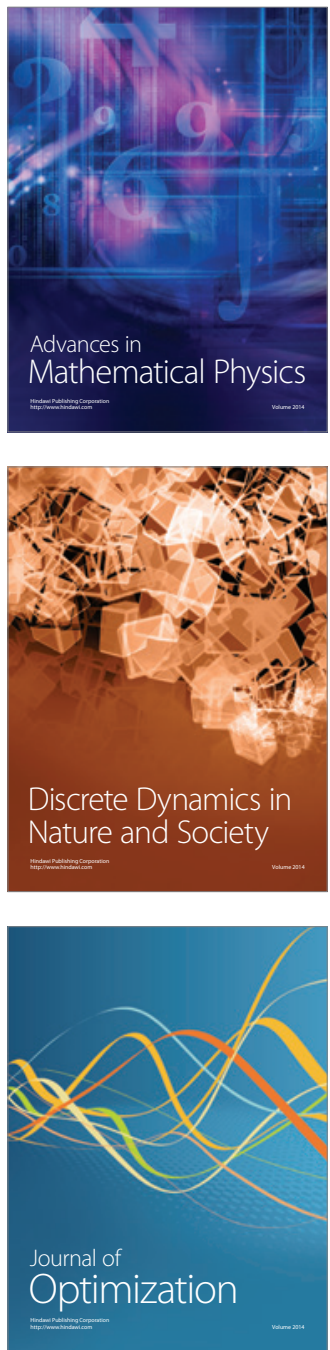